\title{
Silencing of Kruppel-like factor 2 by the histone methyltransferase EZH2 in human cancer
}

\author{
H Taniguchi ${ }^{1,2,3}$, FV Jacinto ${ }^{1,4}$, A Villanueva ${ }^{5}$, AF Fernandez ${ }^{1}$, H Yamamoto $^{2}$, FJ Carmona ${ }^{1}$, \\ S Puertas 5 , VE Marquez ${ }^{6}$, Y Shinomura ${ }^{2}, \mathrm{~K}^{5}$ Imai $^{7}$ and M Esteller ${ }^{1,8,9}$ \\ ${ }^{1}$ Cancer Epigenetics and Biology Program (PEBC), Bellvitge Biomedical Research Institute (IDIBELL), Barcelona, Spain; \\ ${ }^{2}$ First Department of Internal Medicine, Sapporo Medical University School of Medicine, Sapporo, Japan; ${ }^{3}$ Tumor Medical \\ Examination and Treatment Center, Sapporo Medical University School of Medicine, Sapporo, Japan; ${ }^{4}$ Salk Institute for Biological \\ Studies, Molecular and Cell Biology Laboratory, La Jolla, CA, USA; ${ }^{5}$ Catalan Institute of Oncology (ICO), Bellvitge Biomedical \\ Research Institute (IDIBELL), Barcelona, Spain; ${ }^{6}$ Chemical Biology Laboratory, Center for Cancer Research, National Cancer \\ Institute-Frederick, National Institute of Health, Frederick, MD, USA; ${ }^{7}$ The Institute of Medical Science, The University of Tokyo, \\ Tokyo, Japan; ${ }^{8}$ Department of Physiological Sciences II, School of Medicine, University of Barcelona, Barcelona, Spain and \\ ${ }^{9}$ Institucio Catalana de Recerca i Estudis Avançats (ICREA), Barcelona, Spain
}

\begin{abstract}
The Kruppel-like factor (KLF) proteins are multitasked transcriptional regulators with an expanding tumor suppressor function. KLF2 is one of the prominent members of the family because of its diminished expression in malignancies and its growth-inhibitory, pro-apoptotic and anti-angiogenic roles. In this study, we show that epigenetic silencing of KLF2 occurs in cancer cells through direct transcriptional repression mediated by the Polycomb group protein Enhancer of Zeste Homolog 2 (EZH2). Binding of EZH2 to the $5^{\prime}$-end of KLF2 is also associated with a gain of trimethylated lysine 27 histone $\mathrm{H3}$ and a depletion of phosphorylated serine 2 of RNA polymerase. Upon depletion of EZH2 by RNA interference, short hairpin RNA or use of the small molecule 3-Deazaneplanocin $A$, the expression of KLF2 was restored. The transfection of KLF2 in cells with EZH2-associated silencing showed a significant anti-tumoral effect, both in culture and in xenografted nude mice. In this last setting, KLF2 transfection was also associated with decreased dissemination and lower mortality rate. In EZH2depleted cells, which characteristically have lower tumorigenicity, the induction of KLF2 depletion 'rescued' partially the oncogenic phenotype, suggesting that KLF2 repression has an important role in EZH2 oncogenesis. Most importantly, the translation of the described results to human primary samples demonstrated that patients with prostate or breast tumors with low levels of KLF2 and high expression of EZH2 had a shorter overall survival.
\end{abstract}

Oncogene (2012) 31, 1988-1994; doi:10.1038/onc.2011.387; published online 5 September 2011

Keywords: epigenetics; histone methyltransferase; EZH2; KLF2

Correspondence: Dr M Esteller, Cancer Epigenetics and Biology Program (PEBC), Bellvitge Biomedical Research Institute (IDIBELL), 3rd Floor, Hospital Duran i Reynals, Avda. Gran Via de L'Hospitalet 199-203, Barcelona, Catalonia 08908, Spain.

E-mail: mesteller@idibell.cat

Received 5 March 2011; revised 11 July 2011; accepted 28 July 2011; published online 5 September 2011
The Kruppel-like factor (KLF) family of proteins, also known as SP1-like, is made up of a set of transcription factors that are present in a wide range of organisms, in which they fulfill a range of cell differentiation and proliferation functions (Black et al., 2001; Kaczynski et al., 2003; Zhao and Meng, 2005; Bureau et al., 2009). KLFs have Cys2/His2 zinc-finger domains that preferentially bind to GC-rich target sequences, where they can function as activators or repressors in a cell typeand promoter-dependent manner (Black et al., 2001; Kaczynski et al., 2003; Zhao and Meng, 2005; Bureau et al., 2009). KLFs are also emerging as potential tumor suppressor genes owing to their roles in the inhibition of proliferation, migration and angiogenesis, and in the induction of apoptosis, senescence and adhesion (Black et al., 2001; Kaczynski et al., 2003; Zhao and Meng, 2005; Bureau et al., 2009). One member of the family, KLF2, is particularly interesting. Although it is not known to undergo genetic disruption in human tumors, KLF2 expression is diminished in many malignancies, such as prostate (Duhagon et al., 2010) and ovarian (Wang et al., 2005) cancer. From a functional standpoint, KLF2 possesses tumor-suppressor features such as induction of cell quiescence (Buckley et al., 2001), enhancement of DNA-damage-associated apoptosis (Wang et al., 2005), inhibition of leukemia cell proliferation (Wu and Lingrel, 2004), anti-angiogenesis properties (Bhattacharya et al., 2005), and the suppression of cell growth mediated by KRAS (FernandezZapico et al., 2010) and epidermal growth factor receptor (Kannan-Thulasiraman et al., 2010). Thus, we wondered about the molecular basis of the lossof-function defects of KLF2 in human tumorigenesis and so set out to study the possible role of oncogenic repressive mechanisms.

One of the most important systems for maintaining the heritable repression of genes is that of the Polycomb group proteins (Ringrose and Paro, 2004; Martin and Zhang, 2005). These Polycomb group proteins are often de-regulated in human cancer (Pasini et al., 2004; Valk-Lingbeek et al., 2004). The Enhancer of Zeste 
Homolog 2 (EZH2) is a component of the Polycomb repressive complex 2, which also includes SUZ12 and EED, and represses gene transcription by trimethylation of Lys27 of histone H3 (H3K27) (Simon and Lange, 2008). EZH2 has the hallmarks of an oncogene, particularly in prostate and breast cancer, where elevated levels are found in the more advanced forms of the disease (Varambally et al., 2002; Bracken et al., 2003; Kleer et al., 2003). Recently, EZH2 gain-offunction mutations have also been found in lymphomas (Yap et al., 2011). Thus, one way by which EZH2 could promote transformation is by repressing tumor suppressor genes, exploiting its methyltransferase activity for lysine 27 of histone H3 (H3K27) (Simon and Lange, 2008). In this scenario, several genes with a growthinhibitory function have recently been found to be targeted by EZH2 in cancer cells (Chen et al., 2005;
Beke et al., 2007; Yu et al., 2007, 2010; Fujii et al., 2008). Thus, we examined whether KLF2 could also be a key tumor suppressor gene targeted for repression by EZH2 in human tumorigenesis.

To assess the putative role of EZH2 in KLF2 repression, we first transiently depleted the expression of EZH2 by RNA interference (RNAi) in MDA-MB231 and MCF-7 (breast), PC3 and LNCaP (prostate), and U2OS (osteosarcoma) cancer cell lines (Figures 1a and $b$ ). All cell lines were purchased from the American Type Culture Collection (Manassas, VA, USA) and were cultured in Dulbecco's modied Eagle's medium supplemented with $10 \%$ fetal bovine serum. The in vitro transient transfection of short interfering RNAs (Qiagen, Valencia, CA, USA) was performed using Oligofectamine (Invitrogen, Carlsbad, CA, USA). We observed that the loss of EZH2 was associated with a
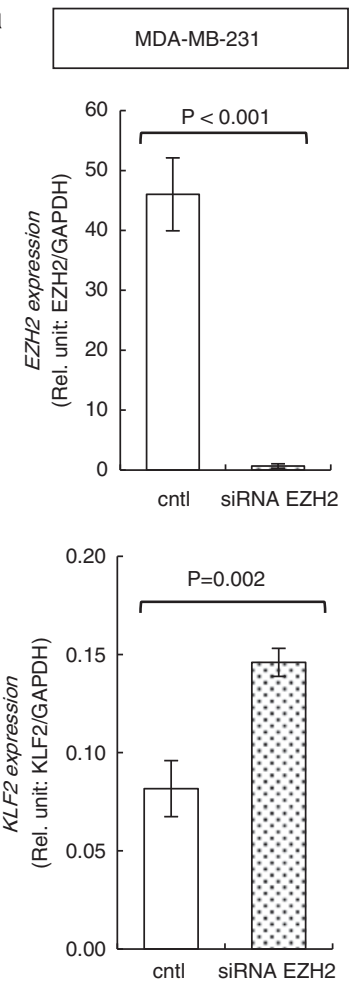
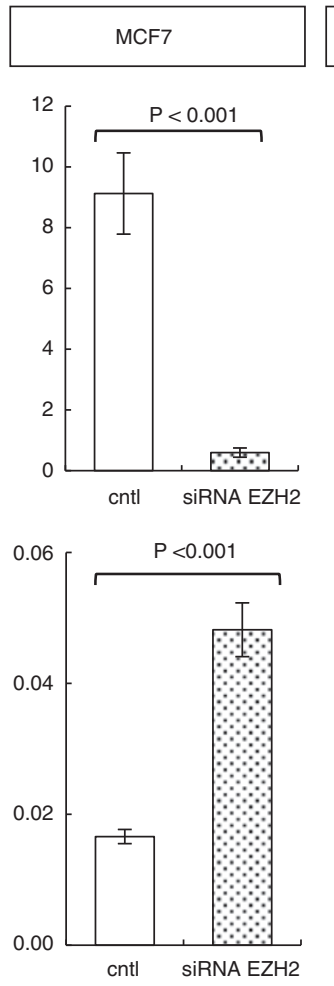
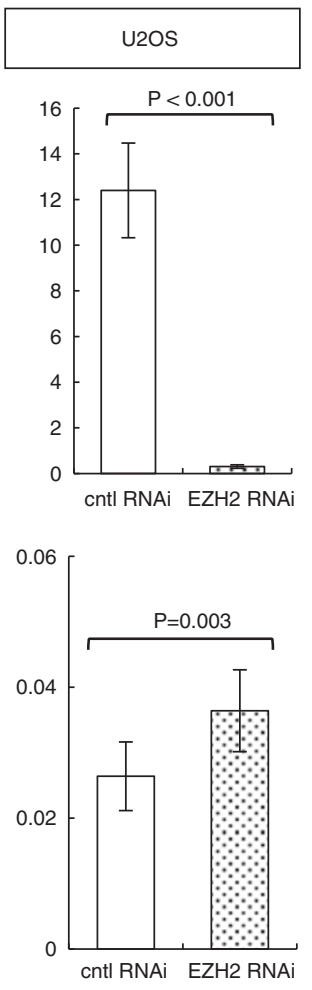
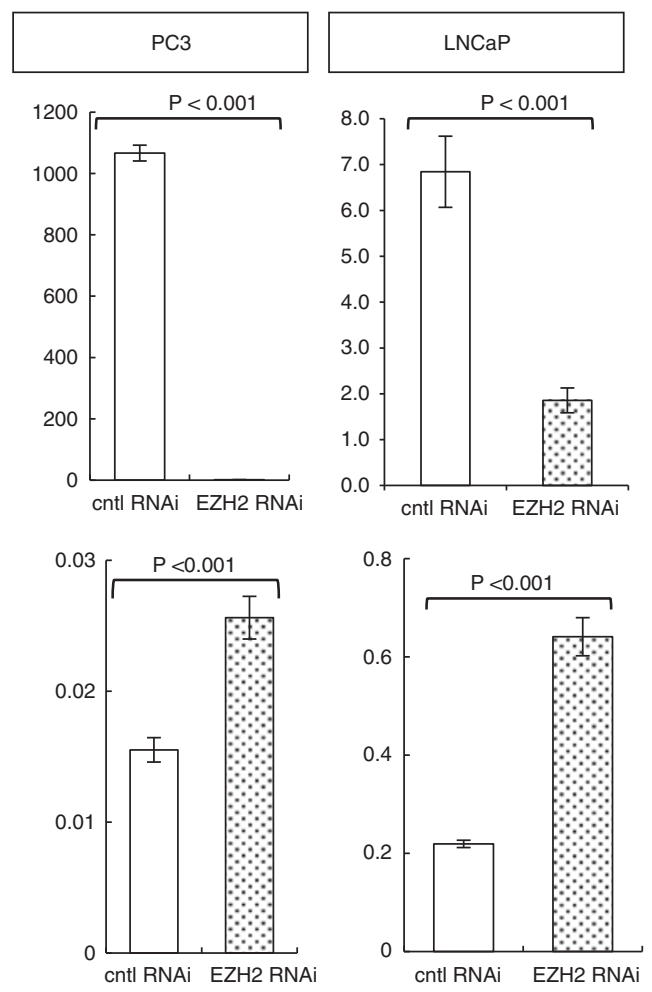
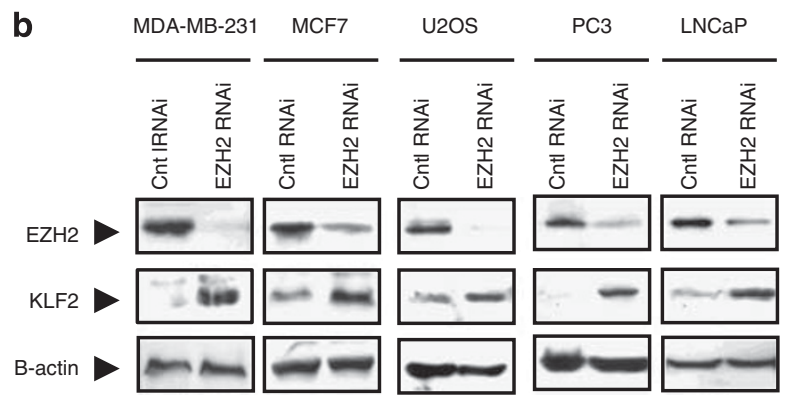

C
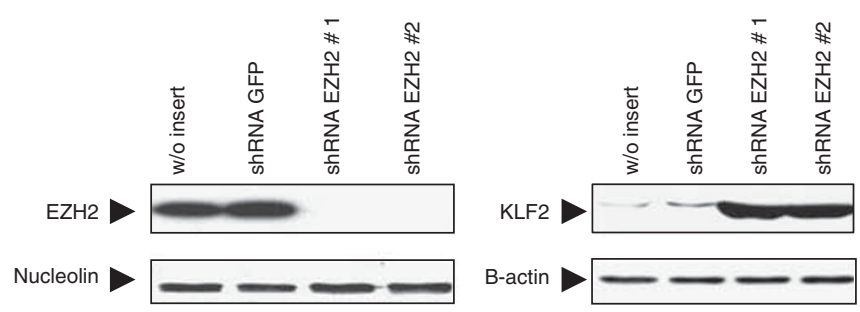

Figure 1 EZH2 depletion leads to increased KLF2 mRNA and protein levels. Expression of KLF2 and EZH2 determined by qRT-PCR (a) and (b) immunoblot in five cancer cell lines (U2OS, MDA-MB-231, MCF-7, PC3 and LNCaP cells) following transfection with oligo-type short interfering RNA (siRNA) against EZH2 or scrambled siRNA for $72 \mathrm{~h}$ as a transient model. (c) Immunoblot of EZH2 and KLF2 in stable EZH2 knockdown clones. U2OS cells are transfected with shRNA constructs targeting EZH2 or control vector and undergo selection with puromycin. $P$-values obtained from Student's $t$-test. 
upregulation of KLF2 at mRNA (Figure 1a) and protein (Figure 1b) levels in all the five described cancer cell lines. Upon EZH2 knockdown at $72 \mathrm{~h}$, we also observed a defect on cell viability determined by the 3(4,5-dimethyl-2-thiazolyl)-2,5-diphenyl-2H-tetrazolium bromide (MTT) assay and an increase in G1/decrease in S-phase assessed by fluorescence-activated cell sorting analysis (Supplementary Figure 1). We strengthened the link between EZH2 expression and KLF2 repression by establishing a U2OS cell line stably transfected with a short hairpin RNA (shRNA) against EZH2 (Figure 1c and Supplementary Figure 2). EZH2-shRNA (Origene, Rockville, MD, USA) transfection was accomplished by electroporation and cells were selected with puromycin (Calbiochem, Darmstadt, Germany). The stable inhibition of EZH2 expression also led to a marked increase in
KLF2 mRNA (Supplementary Figure 2) and protein levels (Figure 1c).

The observed inverse association between EZH2 and KLF2 levels might be mediated by a direct effect of $\mathrm{EZH} 2$ on the KLF2 promoter or by secondary mechanisms. Thus, we performed quantitative chromatin immunoprecipitation (qChIP) for the minimal promoter of KLF2 using antibodies against EZH2, the trimethylated H3K27 mark (3meH3K27) established by the enzyme and phosphorylated serine 2 of RNA polymerase (RNAP-S2, a marker of active transcription). A nonspecific IgG antibody was used as a technical negative control, the EZH2-target gene $A D R B 2$ (Yu et al., 2007) was used as a positive control and the GAPDH locus was used as a negative control. Measurements were made in triplicate and
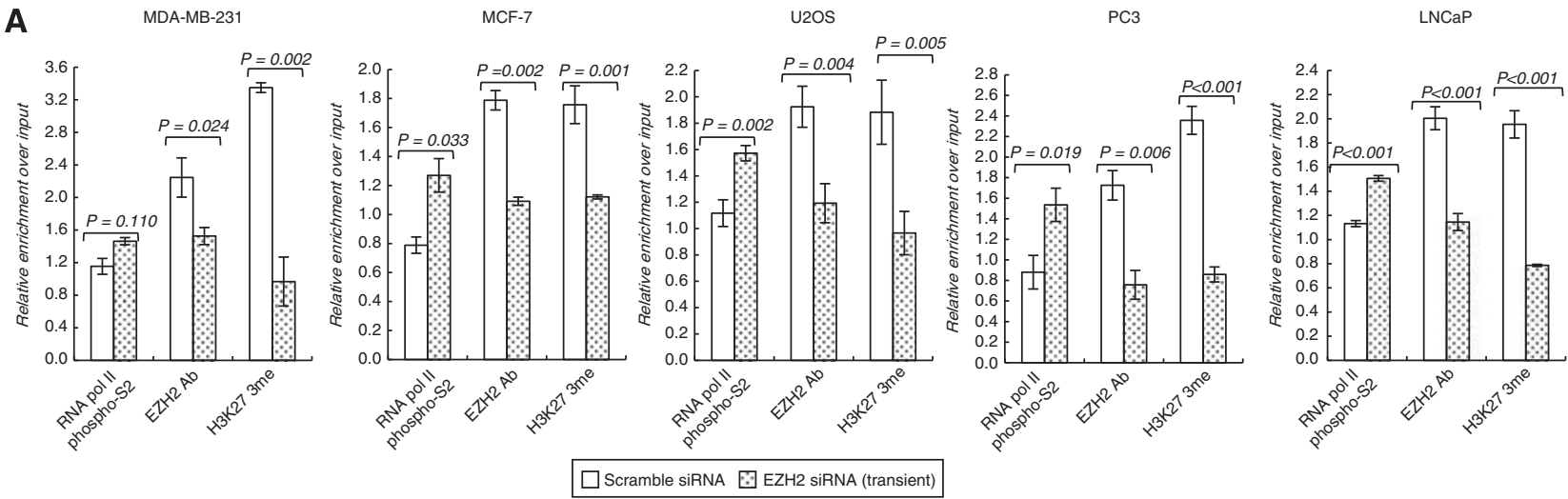

B

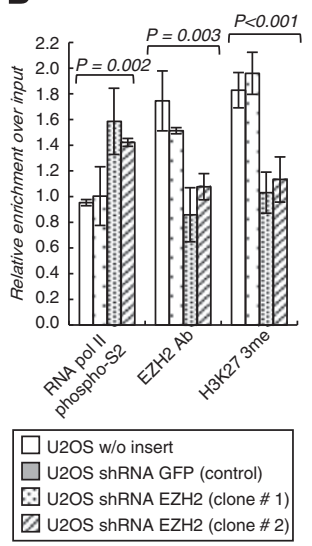

C

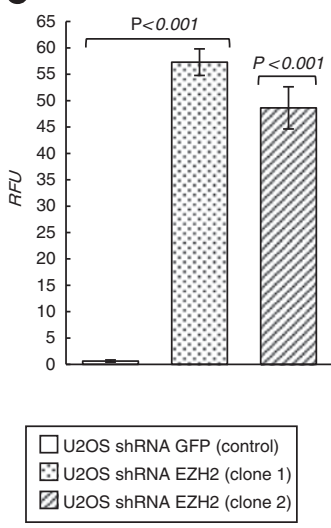

D
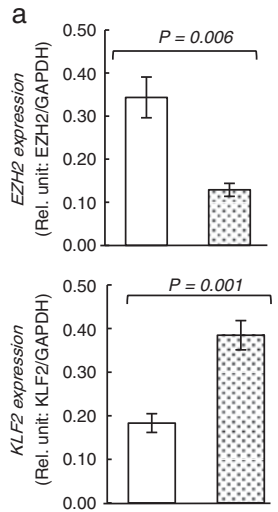

$\square$ Control U2OS

DZNep treated U2OS b
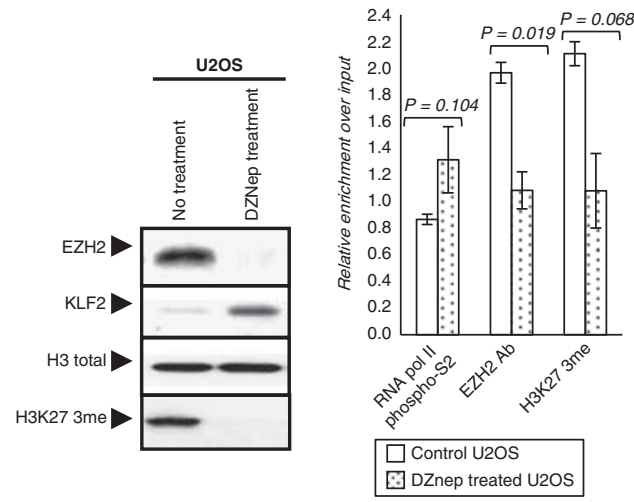

Figure 2 EZH2 direct binding to the KLF2 promoter mediates the transcriptional repression effect. (A) ChIP-qPCR of EZH2 occupancy and H3K27-3me marks in the KLF2 promoter in five cancer cell lines treated with EZH2 siRNA (72 h) or scrambled siRNA. Treatment with siRNA against EZH2 prevents EZH2 occupancy and the presence of the H3K27-3me mark, while enhanced RNAP binding is observed in the KLF2 promoter. ChIP was performed using polyclonal antibodies raised in rabbit against EZH2 (pAb-039-050, Diagenode, Liège, Belgium), RNAP-S2 (ab5095, Abcam, Cambridge, UK) and H3K27me3 (pAB-069-05, Diagenode), with rabbit IgG as a control (ab37415, Abcam, ChIP grade). The primers used for the ChIP-qPCR analysis of the KLF2 promoter were 5'-GAGACTCCAGACT TCCCATCC-3' (sense) and 5'-CAGAGACTCTCAGGGGAGCAC-3' (antisense). (B) qChIP for EZH2 occupancy and H3K27-3me presence for the $K L F 2$ promoter in stable EZH2 knockdown clones (U2OS-shEZH2). (C) $K L F 2$ promoter activities are analyzed by luciferase reporter assay in stable EZH2 knockdown clones. In each experiment, firefly luciferase activities are normalized against those of Renilla. $n=3$, mean \pm s.e.m. (error bars). We used a pGL3 Luciferase Reporter Vector (Promega, Madison, WI, USA) for the KLF2 promoter encompassing NheI/Hind III sites (from -916 to $+129 \mathrm{bp}$ ). (D) Upregulation of KLF2 transcript (a) and protein (b) upon treatment with $5 \mu \mathrm{M}$ DZNep for $72 \mathrm{~h}$. (c) qChIP analysis shows how the treatment with DZNep decreased EZH2 occupancy and the H3K 27-3me mark in the KLF2 promoter, while it enhanced RNAP-S2 occupancy. $P$-values obtained from Student's $t$-test. 
the polymerase chain reactions (PCRs) were done using the Prism 7900 HT Sequence Detection System (Applied Biosystems, Carlsbad, CA, USA). The qChIP analyses demonstrated an enriched presence of EZH2 and 3 meH3K27 in the KLF2 promoter for the described cancer cell lines (Figure 2A). Conversely, RNAP-S2 was depleted at this locus (Figure 2A). The EZH2 RNAi experiments reduced EZH2 occupancy and 3meH3K27 presence and induced the recruitment of RNAP-S2 for the KLF2 promoter (Figure 2A). These results for the $5^{\prime}$-end of the KLF2 gene were similar to those obtained from the qChIP data of the well-known EZH2-target gene $A D R B 2$ (Yu et al., 2007) (Supplementary Figure 3). The U2OS cells that were stably depleted at EZH2 by
shRNA reproduced this qChIP pattern (Figure 2B). In these cells, evidence for the role of EZH2 in directly repressing KLF2 was reinforced by the results of luciferase assays (Figure 2C). Finally, the link between EZH2 binding to the $5^{\prime}$-end of the KLF2 and its corresponding silencing was corroborated by the use of the small molecule 3-Deazaneplanocin A (DZNep), which depletes the cellular levels of Polycomb-repressive complex 2 components, including EZH2 (Tan et al., 2007). Upon DZNep treatment, a marked increase in KLF2 expression was observed in U2OS cells (Figure 2D). The DZNep-mediated enhancement of KLF2 expression was associated with the depletion of EZH2 occupancy and 3meH3K27 levels and an
A

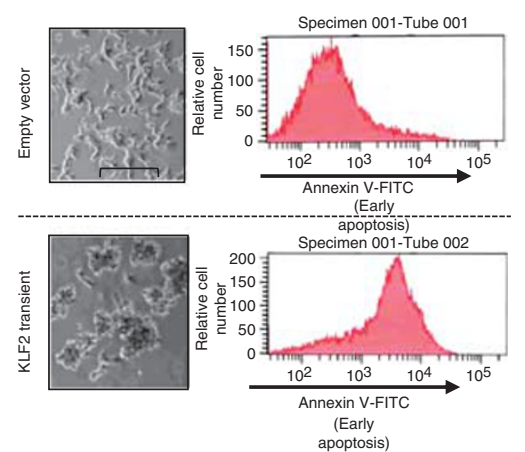

B
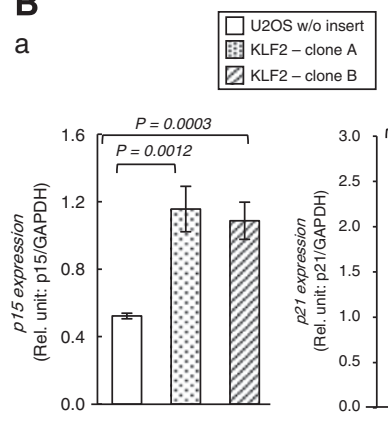
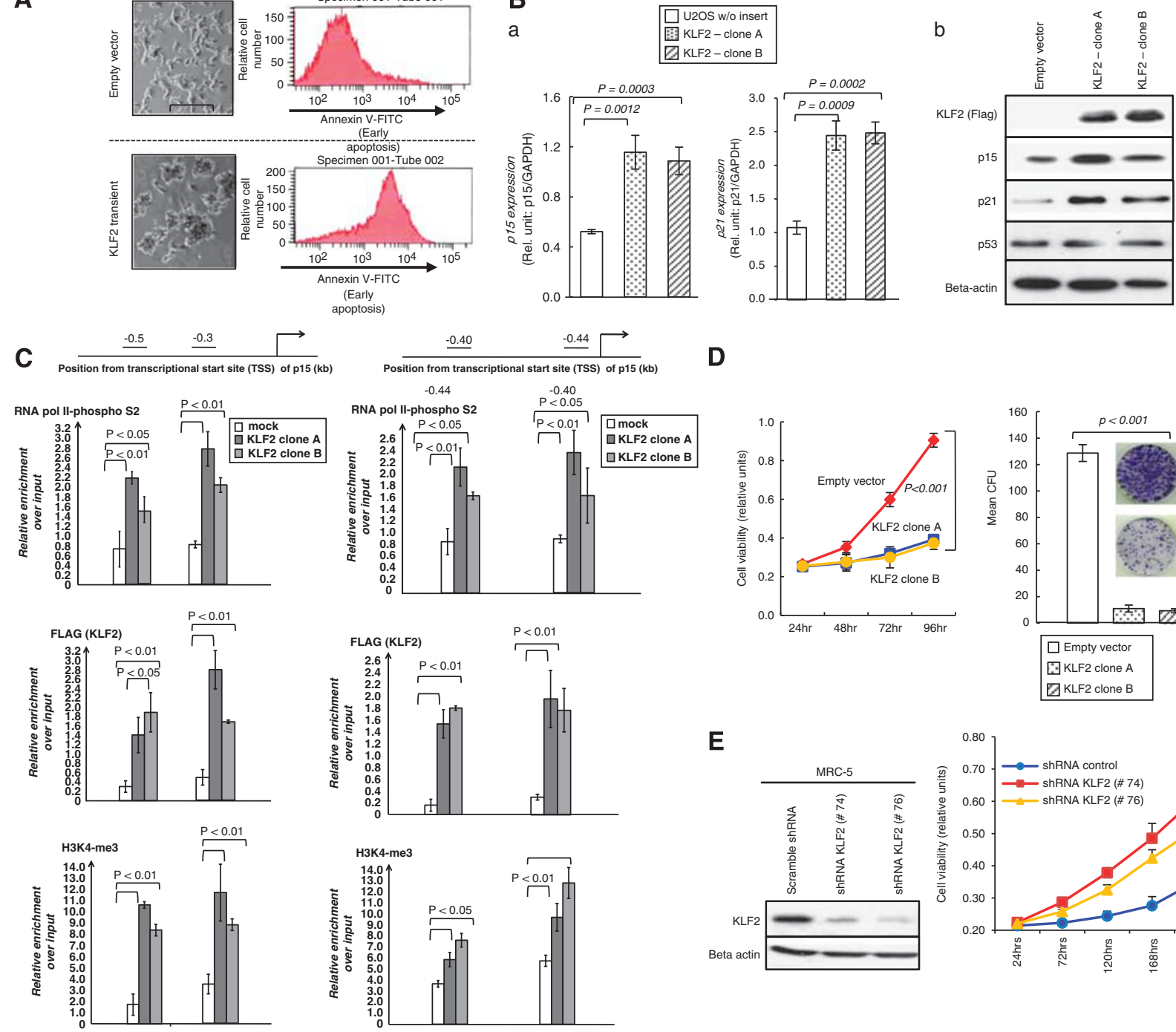

D
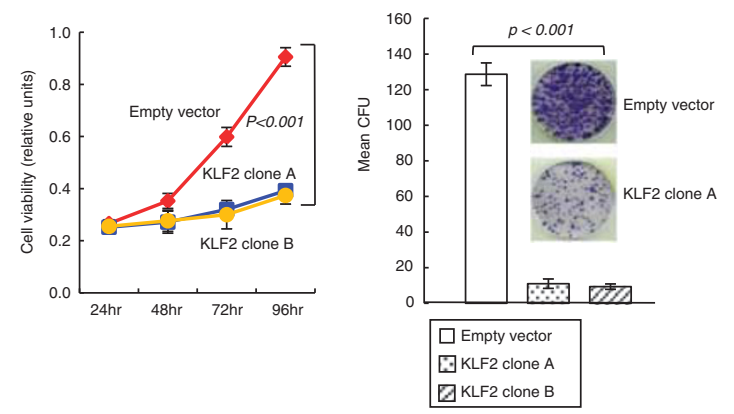

E
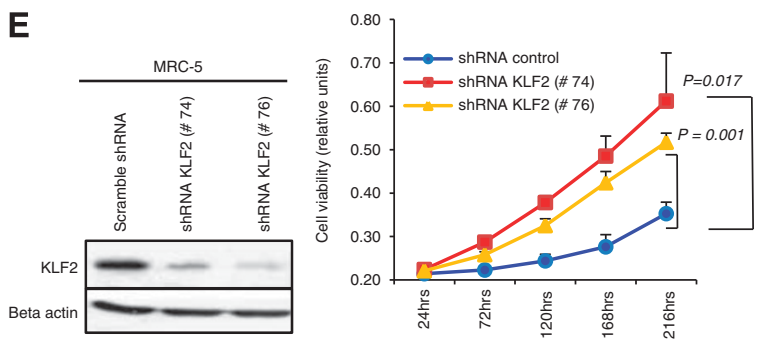

Figure 3 KLF2 induces apoptosis, directly activates the cell cycle-inhibiting genes p15/CDKN2B and p21/CDKN1A and inhibits cell proliferation. (A) Enhanced Annexin V expression relative to empty vector-transfected cells upon KLF2 transfection. The right panels are the original red color/FL-4 (annexin-Cy5) histograms that show how KFL2 transfection induces apoptosis, demonstrated by the increase in the number of cells that incorporate higher amounts of annexin V. (B) U2OS cells transfected with a FLAG-tagged KLF2 (pCMV-Tag2B-KLF2) expression vector show increased expression of $\mathrm{p} 15^{\text {Ink4b }}$ and $\mathrm{p} 21^{\mathrm{CDKN} 1 \mathrm{~A}}$, determined by $\mathrm{qRT}-\mathrm{PCR}$ (a) and western blot (b). (C) The ectopically expressed KLF2 (FLAG-tagged KLF2) occupied the p15 Ink4b and p21 $1^{\text {CDKNIA }}$ promoters in association with a gain of RNAP-S2 occupancy and the H3-K4 trimethylation mark determined by qChIP. (D) MTT (left) and colony formation (right) assays reveal that stable KLF2-expressing U2OS cells grow more slowly than cells transfected with control vector. (E) Enhanced proliferation of normal MRC5 fibroblasts after transfection of shRNA against KLF2 in comparison with scrambled shRNA. $P$-values obtained from Student's $t$-test. 
enrichment of RNAP-S2 in the KLF2 promoter determined by qChIP (Figure 2D). From the DNA methylation standpoint, the KLF 5 '-end region contains a canonical $\mathrm{CpG}$ island that remained unmethylated under all the experimental conditions described (Supplementary Figure 4).

Once we had determined that KLF2 was a direct target of transcriptional repression by $\mathrm{EZH} 2$ in cancer cells, we sought to understand the molecular and cellular contribution of KLF2 silencing to the transformed phenotype. To this end we constructed a FLAG-tagged KLF2 expression vector using the pCMV-Tag2B vector (Stratagene, Santa Clara, CA, USA) and transfected it by electroporation to U2OS cells. Transfected cells were selected by adding G418 (Calbiochem). Upon
KLF2 transfection, we observed enhanced Annexin V expression relative to empty vector-transfected cells (Figure 3A). The pro-apoptotic effect mediated by KLF2 transfection was also associated with an increase in the expression levels of the cell cycle-inhibiting genes p15/CDKN2B and p21/CDKN1A, as determined by qRT-PCR (Figure 3B) and western blot (Figure 3B). These two latter genes could be direct or indirect targets of the transcription factor KLF2. The two possibilities can be discriminated by the chromatin immunoprecipitation assay. The qChIP analyses demonstrated an enriched presence of KLF2 (using a FLAG-M2 antibody) in the $5^{\prime}$-end $\mathrm{CpG}$ islands of p15/CDKN2B and $\mathrm{p} 21 / \mathrm{CDKN} 1 \mathrm{~A}$ in the transfected U2OS cells (Figure 3C). The KLF2 occupancy at these promoters a
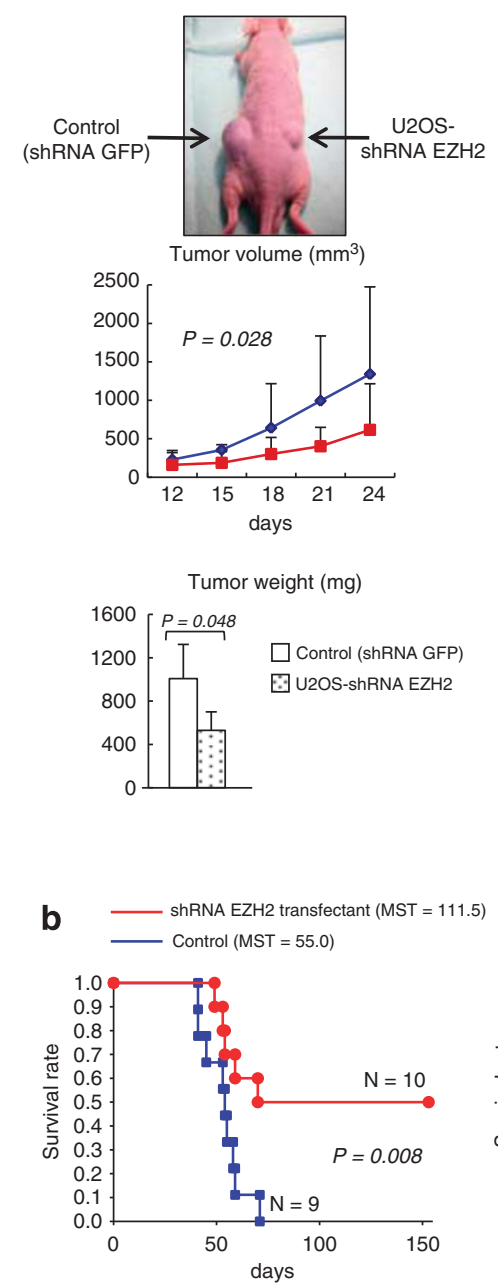
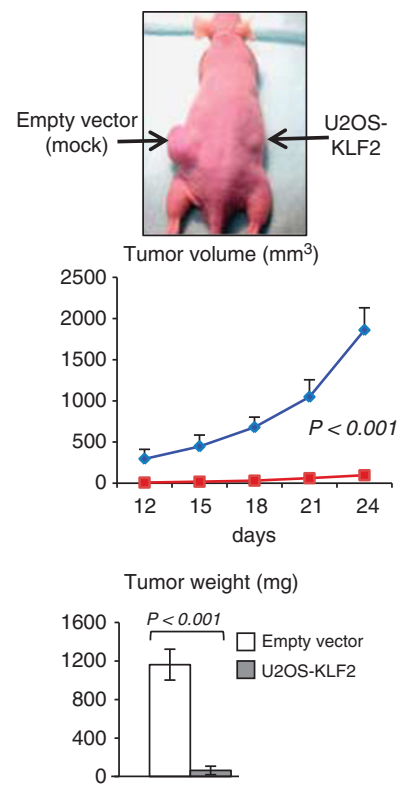

C
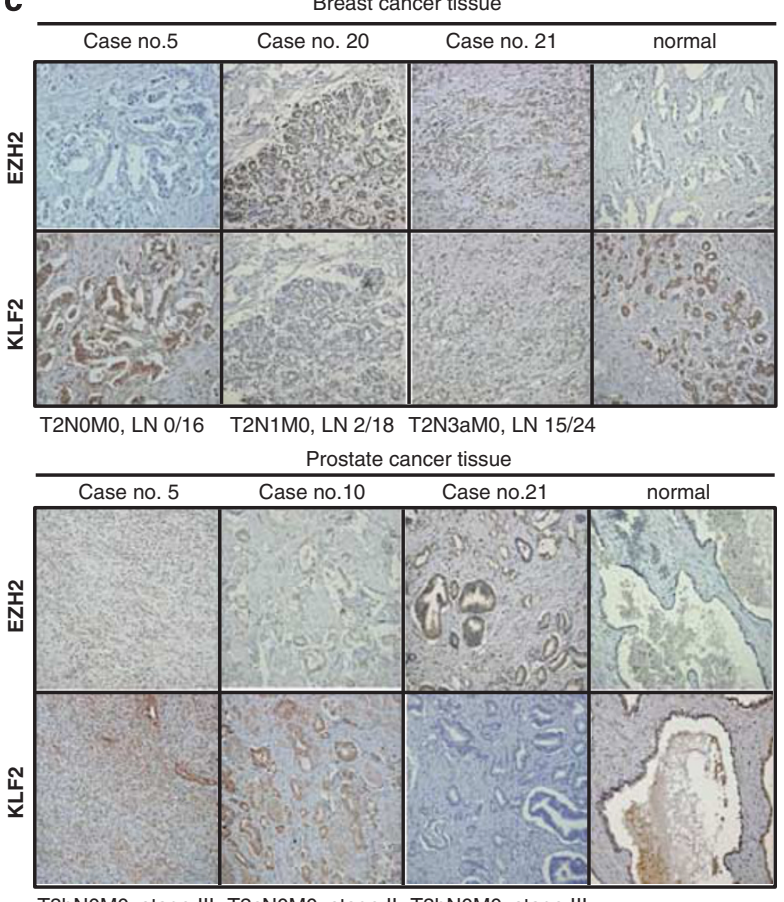

T3bNOM0, stage III T2cNOM0, stage II T3bNOM0, stage III

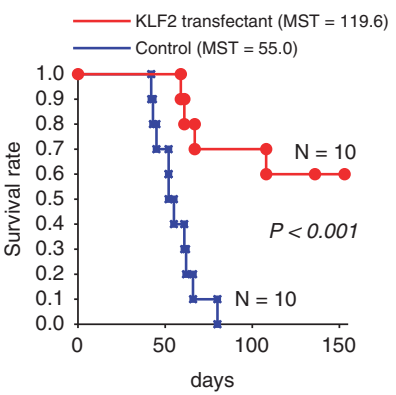

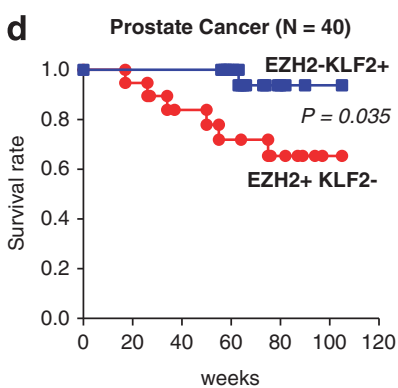

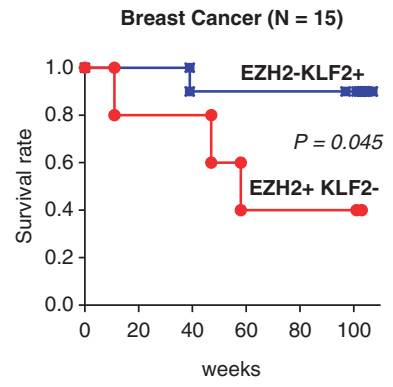

Figure 4 KLF2 as a tumor suppressor in mouse models and its effect on human tumors. (a) Effect of sh-EZH2 knockdown (left) or KLF2 transfection (right) on the growth of U2OS cells inoculated into nude mice. Tumor volume was monitored over time, and the tumor was excised and weighed after 24 days. EZH2 depletion or KLF2 overexpression cause a reduction in tumor volume and weight. (b) Significantly lower mortality following tail-vein injection in the mice of $1 \times 10^{6}$ U2OS cells was observed in U2OS-pCMV-KLF2 or U2OS-shEZH2 cells in comparison with the empty vector-transfected cells $(P<0.001)(\mathbf{c})$ EZH2 and KLF2 expression in clinical cancer samples determined by immunostaining in prostate and breast cancer tissue microarrays. KLF2 expression was inversely associated with EZH2 expression in prostate (Pearson's correlation coefficient $r^{2}=0.32, P<0.05, n=40$ ) and breast (Pearson's correlation coefficient $r^{2}=0.57, P<0.05, n=15$ ) cancer. (d) The high expression of EZH2 associated with the low expression of KLF2 predicts overall shorter survival in breast and prostate cancer (Kaplan-Meier analysis, $P=0.013$ and $P=0.062$, respectively). 
also occurred with an enrichment of active transcription marks, such as RNAP-S2 occupancy and the trimethylation of lysine 4 of histone $\mathrm{H} 3$ (Figure 3C).

From the point of view of cellular growth, KLF2 also had the expected features of a tumor suppressor gene. KLF2-transfected U2OS cells showed a marked reduction of proliferation determined by both the MTT (Figure 3D) and colony-formation (Figure 3D) assays. Conversely, stable depletion of KLF2 by shRNA in the non-transformed MRC5 fibroblast cells increased cell viability as assessed by the MTT assay (Figure 3E). We also wished to investigate the contribution of KLF2 repression to the overall tumorigenic phenotype conferred by EZH2. To do this, we first depleted EZH2 by RNAi in U2OS cells (Supplementary Figure 5) and observed that the induced diminished levels of EZH2 were associated with a lower level of cell proliferation, as determined by the MTT assay (Supplementary Figure 5). This finding was consistent with those of previous reports (Varambally et al., 2002; Bracken et al., 2003). As also described above, EZH2-RNAi caused KLF2 upregulation (Supplementary Figure 5). We proceeded to knock down KLF2 by RNAi in the EZH2-depleted cells to investigate whether the loss of KLF2 was able to 'rescue' partially the oncogenic phenotype mediated by EZH2. We observed that the double RNAi against EZH2 and KLF2 (EZH2-/KLF2cells) gave rise to cells with a higher proliferation rate than those with single EZH2 depletion (Supplementary Figure 5). Thus, KLF2 transcriptional silencing is an important step in the proliferation pathways mediated by the EZH2 oncogene.

We extended the study of the KLF2 growthinhibitory role to in vivo mouse models. Athymic (nu/nu) mice, aged 4-5 weeks, were used for tumor xenograft models. The experimental design was approved by the Bellvitge Biomedical Research Institute Ethical Board. The mice were killed 30 days after injection and tumors were excised and weighed, while the mean volume of tumors \pm standard error of the mean (s.e.m.) was also calculated. The subcutaneous injection of $3 \times 10^{6} \mathrm{U} 2 \mathrm{OS}$ cells in nude mice demonstrated that KLF2-transfected cells (U2OS-pCMVKLF2) developed significantly smaller tumors than empty vector-transfected U2OS cells (Student's $t$-test, $P=0.028$ ) (Figure 4a). The characterization of the spreading potential was developed by tail-vein injection in the mice of $1 \times 10^{6} \mathrm{U} 2 \mathrm{OS}$ cells suspended in $0.2 \mathrm{ml}$ phosphate-buffered saline, and the survival rate at 40 days was analyzed by the Kaplan-Meier method. U2OS-pCMV-KLF2-transfected cells had a significantly lower mortality rate than U2OS empty vector-transfected cells $(P<0.001)$ (Figure $4 b)$. Depletion of EZH2 by shRNA had a similar effect in the reduction of mortality (Figure 4b).

Finally, we decided to translate part of these findings to the context of human primary tumors. We assessed EZH2 and KLF2 expression by immunostaining in clinical tumor samples using a cancer tissue microarray that includes prostate and breast cancer tissue samples with clinical data (SuperBioChips Laboratories, Seoul, Korea). Supplementary Table 1 summarizes the EZH2/ KLF2 expression and clinical data from each individual prostate $(n=40)$ and breast $(n=15)$ cancer case. We observed that KLF2 expression was inversely associated with EZH2 expression in prostate (Pearson's correlation coefficient $r^{2}=0.32, \quad P<0.05$ ) and breast (Pearson's correlation coefficient $\left.r^{2}=0.57, \quad P<0.05\right)$ tumors (Figure 4c). Most importantly, the comparison of the expression data against the clinicopathological values showed that the combination of low KLF2 and high EZH2 expression was associated with shorter overall survival in breast and prostate cancer (Kaplan-Meier analysis, $P=0.013$ and 0.062 , respectively) (Figure $4 d$ ).

Overall, our data indicate that KLF2 undergoes transcriptional silencing in human tumorigenesis by the direct repression of an oncogenic Polycomb protein, the histone methyltransferase EZH2. The EZH2-mediated inactivation of KLF2 blocks the tumor-suppressor features of the KLF2 protein, such as its pro-apoptotic and cell cycle-inhibitory capacities, mediated by $\mathrm{p} 15 / \mathrm{CDKN} 2 \mathrm{~B}$ and $\mathrm{p} 21 / \mathrm{CDKN} 1 \mathrm{~A}$, and its growth-inhibitory features demonstrated in cellular and animal models. Most importantly, the EZH2-mediated loss of KLF2 predicts a poor outcome in human malignancies.

\section{Conflict of interest}

The authors declare no conflict of interest.

\section{Acknowledgements}

We thank O Domínguez for help with the microarray procedures and analysis, F Setien for technical assistance and Miki Kojiya for the flow cytometry analysis. This work was supported by Grants SAF2007-00027-65134, Consolider CSD2006-49, and Lilly Foundation and Dr Josef Steiner Cancer Research Foundation to $\mathrm{ME}$, and the Intramural Research Program of the NIH, National Cancer Institute and Center for Cancer Research to VEM. HT is supported by the Uehara Memorial Foundation and Pancreas Research Foundation of Japan. ME is an ICREA Research Professor.

\section{References}

Beke L, Nuytten M, Van Eynde A, Beullens M, Bollen M. (2007) The gene encoding the prostatic tumor suppressor PSP94 is a target for repression by the Polycomb group protein EZH2. Oncogene 26: $4590-4595$
Bhattacharya R, Senbanerjee S, Lin Z, Mir S, Hamik A, Wang P et al. (2005). Inhibition of vascular permeability factor/vascular endothelial growth factor-mediated angiogenesis by the Kruppel-like factor KLF2. J Biol Chem 280: 28848-28851. 
Black AR, Black JD, Azizkhan-Clifford J. (2001). Sp1 and kruppellike factor family of transcription factors in cell growth regulation and cancer. J Cell Physiol 188: 143-160.

Bracken AP, Pasini D, Capra M, Prosperini E, Colli E, Helin K. (2003). EZH2 is downstream of the pRB-E2F pathway, essential for proliferation and amplified in cancer. EMBO J 22: 5323-5335.

Buckley AF, Kuo CT, Leiden JM. (2001). Transcription factor LKLF is sufficient to program $\mathrm{T}$ cell quiescence via a c-Myc-dependent pathway. Nat Immunol 2: 698-704.

Bureau C, Hanoun N, Torrisani J, Vinel JP, Buscail L, Cordelier P. (2009). Expression and function of Kruppel like-factors (KLF) in carcinogenesis. Curr Genomics 10: 353-360.

Chen H, Tu SW, Hsieh JT. (2005). Down-regulation of human DAB2IP gene expression mediated by polycomb Ezh2 complex and histone deacetylase in prostate cancer. J Biol Chem 280: 22437-22444.

Duhagon MA, Hurt EM, Sotelo-Silveira JR, Zhang X, Farrar WL. (2010). Genomic profiling of tumor initiating prostatospheres. BMC Genomics 11: 324.

Fernandez-Zapico ME, Lomberk GA, Tsuji S, Demars CJ, Bardsley MR, Lin YH et al. (2010). Functional family-wide screening of SP/KLF proteins identifies a subset of suppressors of KRAS-mediated cell growth. Biochem J 435: 529-537.

Fujii S, Ito K, Ito Y, Ochiai A. (2008). Enhancer of zeste homologue 2 (EZH2) down-regulates RUNX3 by increasing histone H3 methylation. J Biol Chem 283: 17324-17332.

Kaczynski J, Cook T, Urrutia R. (2003). Sp1- and Kruppel-like transcription factors. Genome Biol 4: 206.

Kannan-Thulasiraman P, Seachrist DD, Mahabeleshwar GH, Jain MK, Noy N. (2010). Fatty acid binding protein 5 and PPARbeta/delta are critical mediators of epidermal growth factor receptor-induced carcinoma cell growth. J Biol Chem 285: 19106-19115.

Kleer CG, Cao Q, Varambally S, Shen R, Ota I, Tomlins SA et al. (2003). EZH2 is a marker of aggressive breast cancer and promotes neoplastic transformation of breast epithelial cells. Proc Natl Acad Sci USA 100: 11606-11611.

Martin C, Zhang Y. (2005). The diverse functions of histone lysine methylation. Nat Rev Mol Cell Biol 6: 838-849.

Pasini D, Bracken AP, Helin K. (2004). Polycomb group proteins in cell cycle progression and cancer. Cell Cycle 3: 396-400.
Ringrose L, Paro R. (2004). Epigenetic regulation of cellular memory by the Polycomb and Trithorax group proteins. Annu Rev Genet 38: 413-443.

Simon JA, Lange CA. (2008). Roles of the EZH2 histone methyltransferase in cancer epigenetics. Mutat Res 647: 21-29.

Tan J, Yang X, Zhuang L, Jiang X, Chen W, Lee PL et al. (2007). Pharmacologic disruption of Polycomb-repressive complex 2-mediated gene repression selectively induces apoptosis in cancer cells. Genes Dev 21: 1050-1063.

Valk-Lingbeek ME, Bruggeman SW, van Lohuizen M. (2004). Stem cells and cancer; the Polycomb connection. Cell 118: 409-418.

Varambally S, Dhanasekaran SM, Zhou M, Barrette TR, KumarSinha C, Sanda MG et al. (2002). The polycomb group protein $\mathrm{EZH} 2$ is involved in progression of prostate cancer. Nature 419: 624-629.

Wang F, Zhu Y, Huang Y, McAvoy S, Johnson WB, Cheung TH et al. (2005). Transcriptional repression of WEE1 by Kruppel-like factor 2 is involved in DNA damage-induced apoptosis. Oncogene 24: 3875-3885.

Wu J, Lingrel JB. (2004). KLF2 inhibits Jurkat T leukemia cell growth via upregulation of cyclin-dependent kinase inhibitor p21WAF1 CIP1. Oncogene 23: 8088-8096.

Yap DB, Chu J, Berg T, Schapira M, Cheng SW, Moradian A et al. (2011). Somatic mutations at EZH2 Y641 act dominantly through a mechanism of selectively altered PRC2 catalytic activity, to increase H3K27 trimethylation. Blood 117: 2451-2459.

Yu J, Cao Q, Mehra R, Laxman B, Yu J, Tomlins SA et al. (2007) Integrative genomics analysis reveals silencing of beta-adrenergic signaling by polycomb in prostate cancer. Cancer Cell 12: 419-431.

Yu J, Cao Q, Yu J, Wu L, Dallol A, Li J et al. (2010). The neuronal repellent SLIT2 is a target for repression by EZH2 in prostate cancer. Oncogene 29: 5370-5380.

Zhao C, Meng A.. (2005). Sp1-like transcription factors are regulators of embryonic development in vertebrates. Dev Growth Differ 47: 201-211.

This work is licensed under the Creative Commons Attribution-NonCommercial-No Derivative

Works 3.0 Unported License. To view a copy of this license, visit http://creativecommons.org/licenses/by-nc-nd/3.0/

Supplementary Information accompanies the paper on the Oncogene website (http://www.nature.com/onc) 